\title{
Minimization of the Ginzburg-Landau energy functional by a Sobolev gradient trust-region method
}

\author{
P. Kazemia ${ }^{a}$ R. J. Renkab, \\ ${ }^{a}$ Department of Mathematics and Computer Science, Ripon College, P. O. Box 248, Ripon, \\ WI 54971-0248 \\ ${ }^{b}$ Department of Computer Science $\mathcal{E}$ Engineering, University of North Texas, Denton, TX \\ 76203-1366
}

\begin{abstract}
We describe a generalized Levenberg-Marquardt method for computing critical points of the Ginzburg-Landau energy functional which models superconductivity. The algorithm is a blend of a Newton iteration with a Sobolev gradient descent method, and is equivalent to a trust-region method in which the trustregion radius is defined by a Sobolev metric. Numerical test results demonstrate the method to be remarkably effective.

keywords: Ginzburg-Landau; gradient system; least squares; LevenbergMarquardt; Sobolev gradient; trust region
\end{abstract}

\section{Introduction}

Any boundary value problem involving PDE's can be treated as the problem of minimizing a sum of squared residuals. In order to avoid squaring the condition number, it is generally necessary to first convert second-order equations to first order; see, e.g., [1, 2, 3]. An exception is the Ginzburg-Landau equations for which there is a naturally occurring energy functional in the form of a sum of squares and involving only first-order derivatives. In contrast to the case of a sum of squared residuals, the Ginzburg-Landau equations are satisfied by critical points of the energy functional that are not necessarily zeros of the functional. We apply a generalization of the Levenberg-Marquardt algorithm to the Ginzburg-Landau equations in two dimensions.

The standard iterative methods for treating nonlinear least squares problems are gradient descent, Gauss-Newton, and Levenberg-Marquardt or an equivalently trust region method; see, e.g., [4]. Gradient descent methods can achieve convergence from arbitrary starting points, but the rate of convergence is linear. Newton and Gauss-Newton methods can achieve a quadratic rate of convergence but generally require a good initial estimate. The Levenberg-Marquardt

Email addresses: parimah.kazemi@gmail.com (P. Kazemi), robert.renka@unt.edu (R. J. Renka)

Preprint submitted to Elsevier

December 14, 2012 
algorithm benefits from the advantages of both by blending the Gauss-Newton method with the method of steepest descent. It consists of a Newton-like iteration for computing a zero of the gradient of the discretized energy functional. In the standard method a diagonal matrix, scaled by a damping factor, is added to the symmetric positive definite Gauss-Newton approximation to the Hessian. As the damping factor increases, the balance is shifted from the Gauss-Newton direction to the negative gradient direction. In addition to shifting the balance between the two search directions, the added term serves to improve the conditioning of the linear systems. In the original Levenberg algorithm the diagonal matrix is chosen to be the identity. In the Levenberg-Marquardt algorithm the diagonal of the Hessian approximation is used instead.

The Sobolev gradient method is a gradient descent method in which a discretization of a Sobolev gradient is used in place of the Euclidean gradient. The iteration then approximates a gradient system in the Sobolev space, in contrast to the standard method which lacks integrity because the Euclidean gradient generally does not approximate an element of the Sobolev space [5]. We recently observed that the Newton and Gauss-Newton directions are weighted Sobolev gradients ([6]). Rather than blend the Gauss-Newton direction with the Euclidean gradient, it makes more sense to blend it with the unweighted Sobolev gradient. Our generalization of the Levenberg-Marquardt method is thus to replace the diagonal matrix by an operator of the form $D^{t} D$, where $D$ is a differential operator. In the equivalent trust-region formulation of the problem, this corresponds to defining the trust-region radius using the Sobolev norm.

The Ginzburg-Landau energy functional in the mixed vortex state is characterized by numerous critical points which evolve slowly and are difficult to simulate numerically. A central theme of [7] is that computed results are sensitive to mesh resolution, and inadequate resolution leads to spurious solutions which are difficult to distinguish from true solutions. It is observed that results reported in the literature are suspect due to insufficient resolution and the absence of a mesh refinement study. We believe that our gradient descent procedure circumvents this difficulty. Because our method achieves a quadratic rate of convergence, we are able to use small tolerances and to very accurately compute critical points of the discretized functional. More specifically, points that might have been mistaken for solutions if computed with a coarser convergence tolerance are bypassed. We are therefore able to reliably and very efficiently compute critical points using relatively low resolution and with no need for mesh refinement studies. The research presented here was in fact largely motivated by the ambiguity of the term 'solution' in this context. Since we are able to find zeros of the gradient of the discretized Ginzburg-Landau functional to within the limits of machine precision, we have removed the ambiguity.

In Section 2 we consider the general nonlinear least squares problem, and place the standard methods in the context of gradient descent methods. We describe the relationship between Levenberg-Marquardt methods and trust-region methods, and show that our generalization arises in a natural way. In Section 3 we apply the method to the Ginzburg-Landau functional and present numerical test results that demonstrate the effectiveness of the generalization. Section 4 
concludes the paper and points to future research.

\section{Gradient descent and trust-region method}

In this section we formulate Gauss-Newton and Levenberg-Marquardt methods as gradient descent methods, similar to the method of steepest descent, but with gradients that arise from different metrics (inner products). We then describe the relationship between our generalization of the LevenbergMarquardt method and a trust-region constrained minimization problem defined by a Sobolev metric.

Suppose that $r: \mathbb{R}^{n} \rightarrow \mathbb{R}^{m}$ is a $C^{1}$ function. The least squares problem is to minimize

$$
E(u)=\frac{1}{2}\|r(u)\|_{\mathbb{R}^{m}}^{2}=\frac{1}{2}\langle r(u), r(u)\rangle_{\mathbb{R}^{m}},
$$

where $\langle\cdot, \cdot\rangle_{\mathbb{R}^{m}}$ denotes the Euclidean inner product in $\mathbb{R}^{m}$. Taking a Fréchet derivative of $E$ gives

$$
E^{\prime}(u) h=\left\langle r^{\prime}(u) h, r(u)\right\rangle_{\mathbb{R}^{m}}=\left\langle h, r^{\prime}(u)^{t} r(u)\right\rangle_{\mathbb{R}^{n}},
$$

where $r^{\prime}(u)^{t}$ denotes the transpose of the Jacobian $r^{\prime}(u)$. Thus the gradient of $E$ at $u$ in the standard Euclidean metric is

$$
\nabla E(u)=r^{\prime}(u)^{t} r(u) .
$$

The autonomous ODE (gradient system)

$$
u^{\prime}(t)=-\nabla E(u(t)),
$$

defines a path along which the energy $E$ is monotonically decreasing. In the limit as $t \rightarrow \infty$, we have a critical point at which the gradient is zero. In many cases, this solution also gives us a zero of $E$. This evolution equation is a continuous gradient descent method in which the gradient comes from a fixed metric, namely the Euclidean metric.

We now consider a metric that depends on the current approximation $u \in$ $\mathbb{R}^{n}$. We can define a variable metric on $\mathbb{R}^{n}$ by

$$
\langle v, w\rangle_{\mathrm{GN}(u)}=\left\langle r^{\prime}(u) v, r^{\prime}(u) w\right\rangle_{\mathbb{R}^{m}} .
$$

When there are positive constants $c_{1}$ and $c_{2}$ such that $c_{1}\|v\|_{\mathbb{R}^{n}} \leq\left\|r^{\prime}(u) v\right\|_{\mathbb{R}^{m}} \leq$ $c_{2}\|v\|_{\mathbb{R}^{n}}$ for all $u, v \in \mathbb{R}^{n}$, the bilinear form $\langle\cdot, \cdot\rangle_{\mathrm{GN}_{(u)}}$ defines an inner product and a norm on $\mathbb{R}^{n}$ that is uniformly equivalent to the standard norm. By the Riesz Representation theorem, for each $u \in \mathbb{R}^{n}$, there is a unique element of $\mathbb{R}^{n}$, denoted by $\nabla_{\mathrm{GN}(u)} E(u)$ so that, for each $h \in \mathbb{R}^{n}$,

$$
E^{\prime}(u) h=\left\langle h, \nabla_{\mathrm{GN}(u)} E(u)\right\rangle_{\mathrm{GN}(u)} .
$$

We now have

$$
E^{\prime}(u) h=\left\langle r^{\prime}(u) h, r^{\prime}(u) \nabla_{\mathrm{GN}_{(u)}} E(u)\right\rangle_{\mathbb{R}^{m}}=\left\langle h, r^{\prime}(u)^{t} r^{\prime}(u) \nabla_{\mathrm{GN}_{(u)}} E(u)\right\rangle_{\mathbb{R}^{n}}
$$


Thus, from (1) and (2),

$$
\nabla_{\mathrm{GN}(u)} E(u)=\left(r^{\prime}(u)^{t} r^{\prime}(u)\right)^{-1} r^{\prime}(u)^{t} r(u)=\left(r^{\prime}(u)^{t} r^{\prime}(u)\right)^{-1} \nabla E(u) .
$$

The system

$$
u^{\prime}(t)=-\nabla_{\mathrm{GN}(u(t))} E(u(t)),
$$

when discretized using a forward Euler scheme for time, is a Gauss-Newton iteration in which the Hessian $\nabla^{2} E\left(u_{n}\right)=r^{\prime}\left(u_{n}\right)^{t} r^{\prime}\left(u_{n}\right)+\sum_{i=1}^{m} r_{i}\left(u_{n}\right) r_{i}^{\prime \prime}\left(u_{n}\right)$ is approximated by $r^{\prime}\left(u_{n}\right)^{t} r^{\prime}\left(u_{n}\right)$ :

$$
u_{n+1}=u_{n}-\Delta t_{n}\left(r^{\prime}\left(u_{n}\right)^{t} r^{\prime}\left(u_{n}\right)\right)^{-1} \nabla E\left(u_{n}\right) .
$$

Now consider the bilinear form on $\mathbb{R}^{n}$ given by

$$
\langle v, w\rangle_{\operatorname{LM}_{(u)}}=\lambda\langle C v, w\rangle_{\mathbb{R}^{n}}+\left\langle r^{\prime}(u) v, r^{\prime}(u) w\right\rangle_{\mathbb{R}^{m}}
$$

where $C$ is a positive definite matrix of order $n$ and $\lambda=\lambda(u)$ is a nonnegative scalar. Note that for $\lambda>0$ this bilinear form defines an inner product on $\mathbb{R}^{n}$, and the norm induced by the inner product is equivalent to the standard Euclidean norm. A calculation similar to the one given in (2) shows that the gradient of $E$ with respect to the inner product in (3) is given by

$$
\nabla_{\mathrm{LM}_{(u)}} E(u)=\left(\lambda C+r^{\prime}(u)^{t} r^{\prime}(u)\right)^{-1} \nabla E(u) .
$$

Hence the gradient system

$$
u^{\prime}(t)=-\nabla_{\operatorname{LM}(u(t))} E(u(t)),
$$

discretized again using the forward Euler scheme, is a generalized LevenbergMarquardt iteration. If $C$ is the identity matrix, the iteration is the classic Levenberg algorithm, and if $C$ is the diagonal of $r^{\prime}(u)^{t} r^{\prime}(u)$, the iteration is the standard Levenberg-Marquardt algorithm. When the damping constant $\lambda$ is large, the influence of the Gauss-Newton term is reduced, and when the damping constant is small, the influence is great. Thus the algorithm is a blend of the steepest descent and Gauss-Newton algorithms. Below we describe a matrix $C$ that is more appropriate than either of the standard choices when $r$ includes a differential operator.

As discussed in [6], the Levenberg-Marquardt algorithm can be cast as a trust region method. The trust region problem is to minimize the quadratic functional

$$
q_{u}(v)=E(u)+\langle v, \nabla E(u)\rangle_{\mathbb{R}^{n}}+\frac{1}{2}\langle v, H(u) v\rangle_{\mathbb{R}^{n}}
$$

subject to the constraint $\|v\| \leq \delta$, where $H(u)$ is the Hessian or an approximation to the Hessian of $E$ at $u$, and $\delta$ is the radius of the trust region. The quadratic model function is assumed to be a good approximation to $E(u+v)$ when $\|v\| \leq \delta$. If $H(u)$ is the Gauss-Newton approximation, and the constraint region is defined by the norm $\|v\|=\|v\|_{C}=\sqrt{\langle C v, v\rangle_{\mathbb{R}^{n}}}$, then a solution of the 
trust region subproblem is $v=-\nabla_{\mathrm{LM}_{(u)}} E(u)$, where the gradient is defined by (4) for some $\lambda \geq 0$ implicitly defined by the radius $\delta$. The trust region method is thus equivalent to a steepest descent iteration with step-size 1 and the Levenberg-Marquardt gradient. Of course, the actual Hessian may be used as the value of $H(u)$ and in place of the approximate Hessian $r^{\prime}(u)^{t} r^{\prime}(u)$ in (4). In fact, for problems like the Ginzburg-Landau functional, where the residual is not small at critical points, it is necessary to use the actual Hessian in order to achieve a quadratic rate of convergence.

An iteration of the descent method consists of computing an approximate solution $v$ to the trust-region subproblem, and then adjusting the radius $\delta$ based on the actual reduction of the energy relative to the reduction predicted by the quadratic model:

$$
\rho=\frac{E(u)-E(u+v)}{q_{u}(0)-q_{u}(v)} .
$$

In our implementation we use the heuristic method of Nocedal and Wright (Algorithm 4.1 in [4]) in which $\delta$ is reduced to $\|v\|_{C} / 4$ if $\rho<.25$, and $\delta$ is doubled (within an upper bound constraint) if $\rho>.75$ and $\|v\|_{C}=\delta$ (the constraint is active). The current iterate $u$ is updated to $u+v$ if $\rho$ exceeds a small tolerance which we take to be $10^{-4}$.

Now consider the least squares problem in a function space with the $L^{2}(\Omega)$ norm instead of the Euclidean norm, and suppose that in place of $r(u)$ we have $r(D u)$, where $D$ is a differential operator that involves derivatives of order zero and one. Then the permissible functions in the domain of the energy functional are those that are not just square integrable, but that also have square integrable first derivatives, and a natural choice for the norm used in the trust-region constraint is the Sobolev norm for $H^{1}(\Omega)$ defined by

$$
\langle v, w\rangle_{H^{1}(\Omega)}=\langle D v, D w\rangle_{L^{2}(\Omega)}=\langle v, w\rangle_{L^{2}(\Omega)}+\langle\nabla v, \nabla w\rangle_{L^{2}(\Omega)} .
$$

By choosing this norm we exclude from the trust region descent directions for which the first partial derivatives are large as measured in the $L^{2}$ norm. This added constraint ensures that the direction in which we move has additional regularity. In a finite-dimensional discretization in which $D$ maps $\mathbb{R}^{n}$ to $\mathbb{R}^{3 m}$, the $H^{1}$ inner product has the form

$$
\langle v, w\rangle_{H^{1}(\Omega)}=\langle D v, D w\rangle_{\mathbb{R}^{3 m}}=\left\langle\left(D^{t} D\right) v, w\right\rangle_{\mathbb{R}^{n}},
$$

so that $\|v\|_{H^{1}(\Omega)}=\sqrt{\left\langle\left(D^{t} D\right) v, v\right\rangle_{\mathbb{R}^{n}}}$, and the operator $C$ in (4) is $C=D^{t} D=$ $I-\Delta$ for discretized Neumann Laplacian $\Delta=\nabla^{2}$.

We have applied our method to the steady-state Navier-Stokes equations formulated as a first-order least squares system ([8]). In that case we solved the trust-region subproblems by Steihaug's method ([9]). This is a preconditioned conjugate gradient iteration applied to the linear system $H(u) v=-\nabla E(u)$ with early termination if necessary to avoid a constraint violation. The preconditioner is necessarily the same matrix $C$ used to define the trust region. It was found that the Sobolev trust-region method with $C=D^{t} D$ was more efficient 
than the standard trust-region method with $C=\operatorname{diag}(H(u))$ corresponding to Jacobi precondioning. We would expect similar results for the Ginzburg-Landau functional, but we chose instead to implement a dogleg method. The solution $v(\delta)$ to the constrained minimization problem parameterized by $\delta$ is a curved path from 0 to the Newton or Gauss-Newton direction $v_{N}=-H(u)^{-1} \nabla E(u)$. The idea of the dogleg method is to approximate this path by the dogleg path consisting of a line segment from 0 to $v_{S}$ and a line segment from $v_{S}$ to $v_{N}$, where $v_{S}$ is the unconstrained minimizer of $q_{u}$ in the (Sobolev) steepest descent direction:

$$
v_{S}=-\frac{\left\langle\nabla_{S} E(u), \nabla E(u)\right\rangle_{\mathbb{R}^{n}}}{\left\langle\nabla_{S} E(u), H(u) \nabla_{S} E(u)\right\rangle_{\mathbb{R}^{n}}} \nabla_{S} E(u)
$$

with $\nabla_{S} E(u)=\left(D^{t} D\right)^{-1} \nabla E(u)$ denoting the discretized Sobolev gradient. The intersection of the dogleg path with the trust-region boundary is easily computed as the larger of the two roots of a quadratic equation.

In the case of a problem for which a zero residual is sought there may be a gain in efficiency obtained by switching from Gauss-Newton to Newton iterations in the vicinity of the solution, especially when a small tolerance is used to define convergence. We took advantage of this in our treatment of the NavierStokes equations. However, we could discern little visible difference between solutions computed with a small tolerance and those computed with a large tolerance. For the Ginzburg-Landau equations, on the other hand, as discussed in the introduction, it is essential to use a small tolerance in order to distinguish between critical points and spurious solutions. Also, due to the large residual, a quadratic rate of convergence requires Newton's method. Furthermore, as discussed in the next paragraph, it is not necessary to approximate the Hessian when it is not positive definite. We therefore use Newton iterations with fallback to a Gauss-Newton iteration only if the Newton search direction is uphill (has positive inner product with the Euclidean gradient) - a very rare occurrence in our experience. Our tests revealed that efficiency was optimized by this minimal use of the Gauss-Newton search direction.

One of the advantages of Steihaug's method is that a positive definite Hessian is not required. If a direction of negative curvature is encountered, that direction is followed to the trust-region boundary, and the intersection with the boundary is returned as the solution. We extended this idea to the dogleg method. The Newton direction $v_{N}=-H(u)^{-1} \nabla E(u)$ is computed by a preconditioned conjugate gradient method which is simply terminated immediately if a direction of negative curvature is encountered. The resulting value of $v_{N}$ is treated in the same way as a value for which the conjugate gradient method converged. In our testing the updated value $u+v$ obtained from such $v_{N}$ values was sometimes acceptable and sometimes not.

A disadvantage of Steihaug's method is that the initial estimate must be the zero vector. Our method uses the solution from the previous descent step as initial estimate for each subsequent step unless the previous step failed to converge due to a negative curvature direction, in which case we reset the initial estimate to the zero vector. 


\section{Ginzburg-Landau energy functional}

The Ginzburg-Landau model describes the phenomenon of superconductivity which characterizes certain materials that exhibit perfect conductivity and perfect diamagnetism [10]. For a two-dimensional domain $\Omega$, the Gibbs free energy in nondimensionalized form is given by

$$
G(\psi, A)=\frac{1}{2} \int_{\Omega}|\nabla \psi-i A \psi|^{2}+\left|\nabla \times A-H_{0}\right|^{2}+\frac{\kappa^{2}}{2}\left(|\psi|^{2}-1\right)^{2},
$$

where $\psi$ is a complex-valued order parameter whose squared modulus is the density of superconducting electrons, $A$ is the vector potential of an induced magnetic field, $H_{0}$ is an external magnetic field (in the direction normal to the plane of $\Omega$ ), and $\kappa$ is a constant, referred to as the Ginzburg-Landau parameter. It was shown in [11] that, provided $\Omega$ has sufficiently regular boundary, $G$ is well defined for $\psi$ and $A$ in $H^{1}(\Omega)$.

Three types of solution are possible, depending on the values of $H_{0}$ and $\kappa$. In the normal state $\psi=0$ and $\nabla \times A=H_{0}$ so that $G=\int_{\Omega} \kappa^{2} / 4$. In the pure superconducting state $|\psi|^{2}=1, \nabla \psi=0$, and $A=0$ so that $G=$ $\int_{\Omega}\left|H_{0}\right|^{2} / 2$. For $\kappa>1 / \sqrt{2}$ and $H_{0}$ between a pair of positive critical values, the material exhibits what is referred to as a Type II mixed state characterized by superconductivity almost everywhere but with a finite number of vortices of circulating supercurrent (quantized flux) with $\psi=0$ at the centers and a regular distribution throughout $\Omega$. The maximum number of vortices is proportional to the total flux $\int_{\Omega} H_{0}$ so that the size of $\Omega$ is a third parameter that plays a critical role in characterizing solutions.

It is easily verified that the functional $G$, electron density $|\psi|^{2}$, and magnetic field $\nabla \times A$ are all invariant under a gauge transformation defined by

$$
\psi \rightarrow \psi e^{i \chi}, A \rightarrow A+\nabla \chi
$$

for any real-valued function $\chi \in H^{2}(\Omega)$. The Coulomb gauge corresponds to the constraint $\nabla \cdot A=0 \in \Omega$ and $A \cdot \mathbf{n}=0$ on the boundary of $\Omega$ for normal vector $\mathbf{n}$, or equivalently, $A$ is orthogonal (in $L^{2}(\Omega)$ ) to all gradients. This constraint can be enforced weakly by adding the squared norm of $\nabla \cdot A$ to $G$ and projecting $A$ onto the subspace of functions for which $A \cdot \mathbf{n}=0$ on the boundary. When the Coulomb gauge is in effect the only remaining gauge freedom is a constant rotation of the order parameter $\psi$. This freedom can be removed by simply forcing $\operatorname{Im}(\psi)=0$ at a single point for example.

The Euler-Lagrange equations associated with $G(\psi, A)$, referred to as the Ginzburg-Landau equations, have been treated numerically by several methods, including direct solution of the equations with linearization by Newton's method ([12], for example), evolution to steady-state of the time-dependent equations ([7], for example), and minimization of the energy functional by Sobolev gradient descent ([13]). Refer to [14] for a recent review of methods. Here we use the Sobolev trust-region algorithm, which we think of as an enhanced Sobolev gradient descent method, to minimize the functional. In order to achieve a quadratic 
rate of convergence, we use the actual Hessian rather than the Gauss-Newton approximation.

The Sobolev gradient method has been described as a preconditioned forward Euler iteration for evolving the time-dependent equations, but that description obscures an important distinction. Rather than solving the discretized Euler-Lagrange equations, along with boundary conditions, the gradient descent approach is to seek a zero of the Euclidean gradient of the discretized energy functional; i.e., we compute a zero of the gradient of the discretized functional rather than a zero of the discretized gradient. This has the advantage that the nonlinear natural boundary conditions need not be explicitly treated. They are only satisfied by the converged solution rather than being enforced at every iteration. A time-stepping method for the Ginzburg-Landau equations requires careful attention to the boundary conditions as noted in [7]. With the standard Sobolev gradient method it is not necessary to enforce a gauge constraint, but a constraint is necessary for the generalized method because we require an invertible Hessian.

We describe the application of the trust-region algorithm to the GinzburgLandau energy functional in the first subsection, and present test results in Section 3.2 .

\subsection{Trust-region method for Ginzburg-Landau energy}

To put the Ginzburg-Landau energy minimization problem in the context of least squares, let $\Omega$ be a bounded subset of $\mathbb{R}^{2}$ with a sufficiently smooth boundary, and define the following spaces. Let $H=\left[H^{1}(\Omega)\right]^{4}, L_{1}=\left[L^{2}(\Omega)\right]^{12}$, and $L_{2}=\left[L^{2}(\Omega)\right]^{7}$. For $(\psi, A) \in H$, with $\psi=p+i q$ and $A=\left(\begin{array}{l}a \\ b\end{array}\right)$, define $D: H \rightarrow L_{1}$ by $D(\psi, A)=\left(\left(\begin{array}{c}p \\ \nabla p\end{array}\right),\left(\begin{array}{c}q \\ \nabla q\end{array}\right),\left(\begin{array}{c}a \\ \nabla a\end{array}\right),\left(\begin{array}{c}b \\ \nabla b\end{array}\right)\right)$, and define $r: \mathbb{R}^{12} \rightarrow \mathbb{R}^{7}$ by

$$
r(D(\psi, A))=\left(\begin{array}{c}
p_{1}+a q \\
q_{1}-a p \\
p_{2}+b q \\
q_{2}-b p \\
b_{1}-a_{2}-H_{0} \\
\frac{\kappa}{\sqrt{2}}\left(p^{2}+q^{2}-1\right) \\
a_{1}+b_{2}
\end{array}\right)
$$

where the subscripts denote first partial derivatives. With this definition, the Ginzburg-Landau energy with the Coulomb gauge term added is given by

$$
E(\psi, A)=\frac{1}{2}\|r(D(\psi, A))\|_{L_{2}}^{2} .
$$

We reiterate that, while $E$ is expressed as a nonlinear least squares functional, except in some special cases corresponding to a purely superconducting or normal state, we do not seek a zero of $r$. Rather, we are interested in finding solutions $(\psi, A)$ at which the Fréchet derivative of $E$ is zero.

To discretize the problem we take $\Omega$ to be a rectangle, and partition it into a uniform grid of cells. We use second-order central differences to approximate 
first derivatives at cell centers. Function values at cell centers are obtained by averaging the vertex values. The discretized operator $D$ then maps vectors of gridpoint values $v=(p, q, a, b)$ to cell-center values. The integrals $E(\psi, A)$ are approximated by sums of cell-center values scaled by cell areas. Refer to [11] or [13] for further details. Let $H, L_{1}$, and $L_{2}$ now denote the corresponding Euclidean spaces consisting of four vectors of gridpoint values, 12 vectors of cell-center values, and seven vectors of cell-center values, respectively. Then the inner product analogous to $(3)$ with $C=D^{t} D$ is

$$
\langle v, w\rangle_{\mathrm{LM}_{(u)}}=\lambda\left\langle D^{t} D v, w\right\rangle_{H}+\left\langle r^{\prime}(D u) D v, r^{\prime}(D u) D w\right\rangle_{L_{2}}
$$

for $u, v, w \in H$. The gradient of $E$ with respect to this inner product is

$$
\nabla_{\mathrm{LM}_{(u)}} E(u)=\left(\lambda(u) D^{t} D+D^{t} r^{\prime}(D u)^{t} r^{\prime}(D u) D\right)^{-1} \nabla E(u)
$$

where

$$
\nabla E(u)=D^{t} r^{\prime}(D u)^{t} r(D u)
$$

Our computational procedure is the Sobolev trust-region dogleg method described at the end of Section 2. The Hessian and Gauss-Newton approximation to the Hessian are computed by taking partial derivatives of the discretized functional, but then must be modified to enforce the Coulomb gauge constraint. The initial solution estimate $u_{0}$ and the Euclidean gradients $\nabla E(u)$ are projected onto the subspace $V_{0}$ of functions which satisfy the homogeneous boundary conditions $A \cdot \mathbf{n}=0$, and the appropriate rows and columns of the Hessians $H(u)$ are replaced by those of the identity matrix so that the iterates remain in $V_{0}$. While not necessary to ensure invertible Hessians, the gauge freedom is also removed from $\psi$ by including an additional constraint in the definition of $V_{0}$. This was found to consistently improve the efficiency of the descent iteration.

\subsection{Numerical experiments}

For our experiments we took the domain $\Omega$ to be a square with side length 5 , where the units in our nondimensionalization represent penetration depth $\lambda$ of the magnetic field. We discretized the domain with a uniform 65 by 65 grid corresponding to mesh width 5/64 and referred to as the coarse grid. We also employed a refinement of this grid in which the mesh width was halved resulting in 129 by 129 gridpoints. While we could have gained efficiency by using interpolation of coarse-grid solutions as initial estimates for runs on the fine grid, we chose not to do so. All solutions were computed with the constant functions $\psi=1$ and $A=0$ as initial estimates. We tested with $\kappa=4$ and three values of $H 0: 4,6$, and 8 .

We defined convergence by an upper bound of $5 \times 10^{-13}$ on the mean absolute Euclidean gradient component and a bound of $10^{-15}$ on the squared trust-region radius. The first convergence criterion was achieved in most of our tests, but some runs were terminated by the small trust region with the gradient norm as large as $4 \times 10^{-10}$. In no case was there any doubt about the validity of a solution. Table 1 displays the iteration counts, energy values, and gradient 
norms. Tests with the trust region defined by the Euclidean norm (Levenberg method) and with the diagonal of the Hessian (Levenberg-Marquardt method) resulted in iteration counts similar to each other (within a few percentage points) but consistently higher than those associated with the Sobolev trust region (20 to $90 \%)$.

Table 1: Iteration counts, energy values, and errors for $\kappa=4$.

\begin{tabular}{|l|l|l|l|l|}
\hline Grid & $H_{0}$ & Iterations & $E$ & $\|\nabla E\|$ \\
\hline Coarse & 4 & 23 & 44.677 & $4.3 \mathrm{e}-14$ \\
Coarse & 6 & 22 & 55.946 & $1.0 \mathrm{e}-13$ \\
Coarse & 8 & 63 & 67.255 & $4.2 \mathrm{e}-16$ \\
\hline Fine & 4 & 87 & 44.046 & $2.0 \mathrm{e}-16$ \\
Fine & 6 & 28 & 55.845 & $9.2 \mathrm{e}-11$ \\
Fine & 8 & 88 & 65.858 & $6.4 \mathrm{e}-15$ \\
\hline
\end{tabular}

Contour plots of the computed densities $|\psi|^{2}$ are depicted in Figure 1. The top row depicts the degree- 4 critical points computed for $H_{0}=4$ with the higher energy solution on the left, while the bottom row depicts the critical points associated with $H_{0}=6$ (left) and $H_{0}=8$ (right). The degrees (winding numbers) of the last two are 12 and 24, respectively.

The two degree- 4 critical points demonstrate that, even with the same number of vortices, there are multiple solutions. The fact that the lower-energy solution was obtained on the finer grid is purely coincidental. The critical point computed depends on the initial estimate. We have developed a tool that allows us to generate initial estimates that mimic arbitrary distributions of vortices, and thus to systematically study the solution set associated with a given domain and $\left(\kappa, H_{0}\right)$ pair. Some critical points, such as the higher-energy degree- 4 solution, however, appear to be saddle points, and are very easily bypassed even with a good initial estimate. For computing such points we have devised a stable method for finding a zero of $\nabla E(u)$ by minimizing $\phi(u)=\|\nabla E(u)\|^{2} / 2$ using the observation that

$$
\phi^{\prime}(u) h=\left\langle\nabla E^{\prime}(u) h, \nabla E(u)\right\rangle=\left\langle h, \nabla E^{\prime}(u) \nabla E(u)\right\rangle
$$

for all $u, h \in H$, so that

$$
\nabla \phi(u)=\nabla E^{\prime}(u) \nabla E(u) \approx \frac{1}{\delta}[\nabla E(u+\delta \nabla E(u))-\nabla E(u)]
$$

for small $\delta>0$.

\section{Conclusion}

We have described a new method for computing solutions to the GinzburgLandau equations. The method is efficient and robust, achieving quadratic convergence on every problem on which it has been tested. It has neither the 


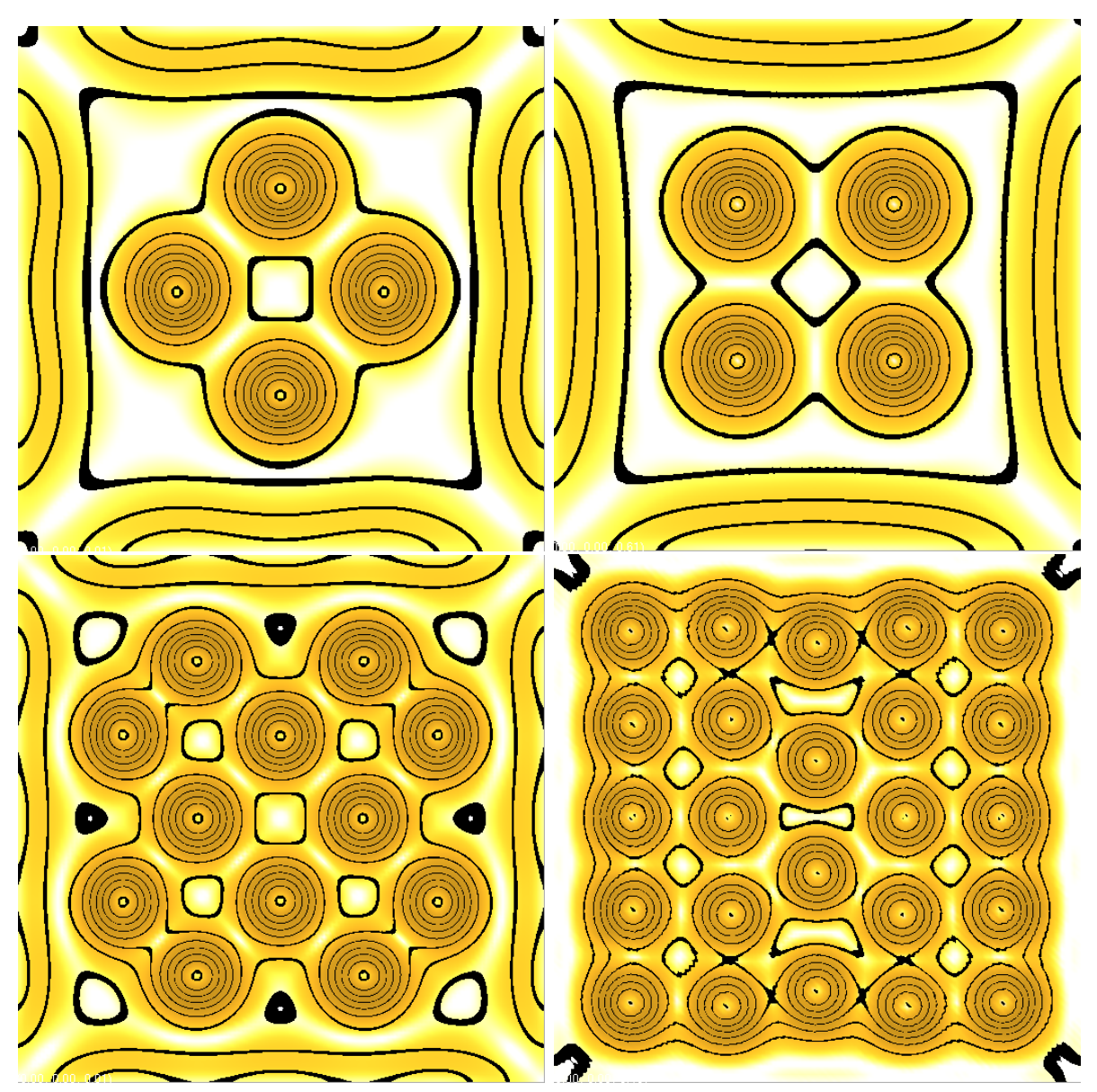

Figure 1: Vortex configurations $\left(|\psi|^{2}\right), \kappa=4, H_{0}=4,4,6$, and 8

need for accurate initial estimates suffered by methods that attempt to solve the discretized Euler-Lagrange equations, nor the difficulty of separating actual solutions from spurious solutions characterizing methods that attempt to evolve the time-dependent equations to steady-state. It provides the basis for a powerful simulation tool that could be used both to advance the science of superconductivity and for engineering analysis and design of superconducting microelectronics.

It is also important to understand the transient and dynamic behavior of superconductors as required, for example, by the experiments described in [15] and [16]. It remains to be seen how successfully our method might be extended to the time-dependent equations. Extension to a three-dimensional domain is very straightforward. It would also be interesting to test the method on multiply-connected domains.

Our method may be thought of as a finite dimensional emulation of a con- 
tinuous gradient system in a Sobolev space in which the gradient is defined by a Sobolev inner product that varies continuously and smoothly over the space. This is similar to a Riemannian manifold in which inner products are assigned to tangent spaces in a differentiable fashion. We have made a start toward extending the theory of Sobolev gradients to variable metric methods in [17], but much work remains to be done.

\section{References}

[1] Z. Cai, R. Lazarov, T. Manteuffel, S. McCormick, First-order system least squares for second-order partial differential equations, SIAM J. Numer. Anal. 31 (1994) 1785-1799.

[2] Z. Cai, T. Manteuffel, S. McCormick, First-order system least squares for second-order partial differential equations, SIAM J. Numer. Anal. 34 (1997) $425-454$.

[3] B. Jiang, The Least-Squares Finite Element Method: Theory and Applications in Computational Fluid Dynamics and Electromagnetics, SpringerVerlag, Berlin, 1998.

[4] J. Nocedal, S. J. Wright, Numerical Optimization, Springer, 1999.

[5] Neuberger J. W., Sobolev Gradients and Differential Equations, 2nd edition, Springer, 2010.

[6] R. J. Renka, Nonlinear least squares and Sobolev gradients, Applied Numerical Mathematics (2012), 10.1016/j.apnum.2012.12.002.

[7] W. B. Richardson, A. L. Pardhanani, G. F. Carey, A. Ardelea, Numerical effects in the simulation of Ginzburg-Landau models for superconductivity, Int. J. Numer. Methods Eng. 59 (2004) 1251-1272.

[8] R. J. Renka, A Sobolev gradient method for treating the steady-state incompressible Navier-Stokes equations, Cent. Eur. J. Math., in press.

[9] T. Steihaug, The conjugate gradient method and trust regions in large scale optimization, SIAM J. Numer. Anal. 20 (1983) 626-637.

[10] Michael Tinkham, Introduction to Superconductivity, second edition, Dover, 2004.

[11] Parimah Kazemi, Using projections in a direct minimization scheme for a wide class of energy functionals, Communications on Applied Nonlinear Analysis 15 (4) (2008) 69-98.

[12] Q. Du, M. Gunzberger, J. S. Peterson, Analysis and approximation of the Ginzburg-Landau model of superconductivity, SIAM Rev. 34 (1992) 54-81. 
[13] J. W. Neuberger, R. J. Renka, Sobolev Gradients and the Ginzburg-Landau Functional, SIAM J. Sci. Comp. 20 (2) (1998) 582-590.

[14] Q. Du, Numerical approximations of the Ginzburg-Landau models for superconductivity, Journal of Mathematical Physics 46095109 (2005).

[15] G. W. Crabtree, G. K. Leaf, H. G. Kaper, V. M. Vinokur, A. E. Koshelev, D. W. Braun, D. M. Levine, W. K. Kwok, J. A. Fendrich, Time-dependent Ginzburg-Landau simulations of vortex guidance by twin boundaries, Physica C 263 (1996) 401-408.

[16] G. W. Crabtree, G. K. Leaf, H. G. Kaper, D. W. Braun, V. M. Vinokur, A. E. Koshelev, Dynamic vortex phases in superconductors with correlated disorder, Preprint ANL/MCS-P590-0496, Mathematics and Computer Science Division, Argonne National Laboratory, 1996.

[17] P. Kazemi, R. J. Renka, A Levenberg-Marquardt method based on Sobolev gradients, Nonlinear Analysis Series A: Theory, Methods \& Applications 75 (2012) 6170-6179, 10.1016/j.na.2012.06.022. 